\title{
Enhancement LTE System Based on DWT and Four STBC Transmit Antennas in Multichannel Models
}

\author{
Laith Ali Abdul-Rahaim \\ Electrical Engineering Department, Babylon University, Babil, Iraq
}

Email address:

drlaithanzy@yahoo.com

To cite this article:

Laith Ali Abdul-Rahaim. Enhancement LTE System Based on DWT and Four STBC Transmit Antennas in Multichannel Models. American Journal of Networks and Communications. Vol. 4, No. 2, 2015, pp. 10-20. doi: 10.11648/j.ajnc.20150402.11

\begin{abstract}
In this paper Enhancement detail for the two main applications of Long Term Evolution (LTE) these are fixed and Mobile LTE. Fixed LTE will send the data from a single point-to-multipoint like user's houses and companies. While full mobility achieved by Mobile LTE to cellular networks. This done at very high broadband data rate comparted with other broadband networks like WiMax and Wi-Fi. The two types of LTE above are used in planning of a proper network which offers better throughput wireless broadband connectivity with lower cost. This work present a new proposed structures for LTE based on Space Time Block Coding (STBC-LTE) and Discrete Wavelets Transform (DWT) as multicarrier. The purpose of these new proposed structures is to improve the performance of bit error rate (BER) compared with the conventional STBC-LTE that use fast Fourier transform (FFT) as multicarrier. In addition, the new proposed structures with more than three transmits antennas and DWT was used in first time in LTE systems to enhance spectral efficiency and supports BER performance. The proposed STBC- LTE systems have been examined under different channel models like "AWGN, flat fading, and three types of multipath selective fading channel models (Extended Pedestrian, Extended Vehicular and Extended Typical Urban)". The simulation results achieved in this work show that STBC- LTE based on DWT with four transmits antennas given best between other conventional STBC-LTE based on FFT systems. All these LTE systems models were built using MATLAB 2014a to permit various system parameters to be changed and tested like signal to noise ratio (SNR) and maximum Doppler shift and type of channel and channel parameters of the system.
\end{abstract}

Keywords: LTE, Multipath Fading Channels, DWT, STBC, OFDM

\section{Introduction}

With the Large increase of wireless networks and multimedia applications such as audio streaming, mobile TV, interactive gaming, video and Internet browsing, the mobile communication technology needs to meet different requirements of mobile data, mobile calculations and mobile multimedia operations. In order to prepare this increasing in mobile data usage and the new applications of multimedia, LTE and (LTE-A advance) technologies have been specified by the 3GPP as the emerging technologies of mobile communication for the next generation wireless broadband mobile networks.

The LTE system is designed to be a packet-based system containing less network elements, which improves the system capacity and coverage, and provides high performance in terms of high data rates, low access latency, flexible bandwidth operation and seamless integration with other existing wireless communication systems [1]. The LTE-A system specified by the 3 GPP LTE Release 10 enhances the existing LTE systems to support much higher data usage, lower latencies and better spectral efficiency [2]. In addition, both of the LTE and LTEA systems support "flat IP connectivity, full interworking with heterogeneous wireless access networks and many new types of base stations such as pico/femto base stations and relay nodes in a macro-cellular network". Due to the introduction of the new characteristics, it incurs a lot of new challenges in security issue with the design architectures of the LTE and LTE-A systems.

The Long-Term Evolution LTE is the newest expansion in the systems of 3 GPP $[3,4]$. In 2004, the beginning of the first study in LTE systems, at this time the LTE was refers to the prospect evolution of UMTS. However, the word "LTE" bewitched many researchers, and so it became the favorite 
name between all new wireless communication systems. "Evolved UTRAN (E-UTRAN)" is name of radio access network in LTE systems as compared with the UTRAN in UMTS. The upgraded 3GPP radio access network from GSM to UMTS needed a little change in core network. However, when the radio access network was upgraded from UMTS to LTE, also would enhanced the core network. "System Architecture Evolution (SAE)" is targeting enhancement the standardization work of the core network architecture. And "the Evolved Packet Core (EPC)" term means the evolved core network. SAE based on an all-IP network and supports the GPP radio access networks and non-3GPP radio access networks like CDMA2000, Wi-Fi and WIMAX. However EPC's support the non-3GPP radio access networks and enables the operations of previous non3GPP radio access networks to espouse LTE as their future radio access network of these networks. The term "Evolved Packet System (EPS)" means E-UTRAN and EPC combination $[4,5]$.

The description to the LTE systems was been defined in Release 8 as the $1^{\text {st }}$ idea of the standard of LTE system that including the basal functionality to perform it as a wireless system. LTE Release 9 makes a major addition by adding "the Multimedia Broadcast/Multicast Service (MBMS)", which provide LTE broadcast and multicast services. The new features that added in Release 10 of LTE will support "Carrier Aggregation (CA)" that encloses multiple LTE carriers used to provide a high speed rate. This additional will increase LTE coverage; and support access from many machine-type devices which called Machine-Type Communication (MTC). In beginning of 2012, "LTE Release 11 standardization" was been published and the Major works LTE Release 11 are the improvement of "MBMS and CA" [6, 7]. At the World Radio communication Conference (WRC07), it was pointed out that there is a strong need to add spectrum for mobile systems due to the expected large increase in next 15 years. The WRC07 defined "new bands several for IMTAdvanced: $450 \mathrm{MHz}$ band; UHF band (698-960 MHz); $2.3 \mathrm{GHz}$ band; C-band (3400-4200 MHz)" [8,9].

\section{The Proposed LTE Structure}

The LTE can implement using Discrete Fourier transform (DFT) or fast Fourier transforms (FFT) as complex exponential functions. So it can be replaced by or discrete wavelets transform (DWT). This replacement will decrease the interference level. From many research, it's found that uses of discrete orthonormal wavelets will reduce the ICI and ISI because the DWT will Strengthens the orthogonality between the subcarrier [10]. The simulation results in $[11,12$, 13] show this idea of replacement FFT by DWT in some multicarrier system and calculate the BER performance with these orthogonal bases. The simulations of LTE system with new transform have shown the dependence of channel on the performance of DWT and FFT. The main idea for using DWT in LTE system is the excellent spectral containment wavelet filters properties over Fourier filters. Under certain channel conditions, it has been found that DWT based LTE does outperform better than FFT based LTE. The implementations of LTE in practice today have been done by using FFT and its inverse operation IFFT (or DWT and its inverse operation IDWT) to represent multicarrier modulation and demodulation. The Intersymbol interference (ISI) can be eliminated almost completely by adding a guard time interval in each packet of LTE frame and this will causes a lose about $25 \%-40 \%$ from data rate and this is one of the disadvantage of FFT-LTE. So the uses of DWT instead of DFT will increase the orthogonality between the subcarrier of the LTE packet and will be combat the narrowband interference and so no need to adding a guard time interval $[14,15]$.

The second proposed idea to LTE system is adding space time blocks coding (STBC) to the system. The STBC reduce the effect of multipath frequency selective Multipath fading channel. The aims this paper are designing a wireless communication system with least bit error rate (BER) for high data rate to fix stationary nodes and mobile users under multichannel models. These ideas will be implemented in LTE system by adding STBC with more than two antennas and using DFT or DWT $[1,16,17]$.

The proposed STBC-LTE transceiver is shown in Fig. (1). All the type of space-time block codes with three transmitters or more has a coding rate of $1 / 2$, to satisfy orthogonality condition. The space-time block code for four transmits antennas $\mathrm{N}=4$, with input symbols $\left(S_{1}, S_{2}, S_{3}, S_{4}\right)$, the output will be over $\mathrm{T}=8$ symbol periods, thus the coding rate $\mathrm{R}$ $=1 / 2[18,19]$. At a given symbol period, four antennas transmitted four signals simultaneously. At time slot T0, transmitted signal from first transmitter $\left(T_{x 1}\right)$ is denoted by $S_{1}$, the signal from second transmitter $\left(T_{x_{2}}\right)$ by $S_{2}$ and the signal from third transmitter $\left(T_{x 3}\right)$ by $S_{3}$ and the signal from fourth transmitter $\left(T_{x 4}\right)$ by $S_{4}$. This process will go on in the same manner for each time slot until transmitting the last row of Table 1. This table has a rate of $(1 / 2)$ and is used as STBC encoder to transmit any complex signal constellations [20,21]. For the four transmit and one receive antenna system, the channel coefficients are modeled by a complex multiplicative distortions, $h_{1}$ for the first transmit antenna, $h_{2}$ for the second transmit antenna and $h_{3}$ for the third transmit antenna. $h_{4}$ for the fourth transmit antenna[22]. Since some models used in this work are time varying and frequency selective for wide band mobile communication systems, so a dynamic estimation of channel is necessary to compensate LTE signal [4]..There are two types of channel estimations, block type and comb-type pilot channel estimation as shown in [15]. After pilot-carrier (training sequence) is generated as a bipolar sequence $\{ \pm 1\}$, the receiver previously knows this sequence. So the system can estimate the channel transfer function $h_{1}(t), h_{2}(t), h_{3}(t)$ and $h_{4}(t)$. The inverse of these channels also will be calculated. The channel transfer function estimation and the inverse of it are applied to each LTE packet to reduce the channel effects and bit errors rate BER, much like equalization [12] 
Table (1). STBC mapping for four transmit antennas using complex signals

\begin{tabular}{lllll}
\hline \multirow{5}{*}{ Time slot } & \multicolumn{4}{l}{ Four transmit antennas } \\
\cline { 2 - 5 } & \multicolumn{4}{l}{ Three transmit antennas } \\
\cline { 2 - 5 } & $\mathbf{T}_{\mathbf{x} 1}$ & $\mathbf{T}_{\mathbf{x} 2}$ & $\mathbf{T}_{\mathbf{x} 3}$ & $\mathbf{T}_{\mathbf{x} 4}$ \\
\hline Slot T0 & $S_{1}$ & $S_{2}$ & $S_{3}$ & $S_{4}$ \\
Slot T1 & $-S_{2}$ & $S_{1}$ & $-S_{4}$ & $S_{3}$ \\
Slot T2 & $-S_{3}$ & $S_{4}$ & $S_{1}$ & $-S_{2}$ \\
Slot T3 & $-S_{4}$ & $-S_{3}$ & $S_{2}$ & $S_{1}$ \\
Slot T4 & $S_{1}^{*}$ & $S_{2}^{*}$ & $S_{3}^{*}$ & $S_{4}^{*}$ \\
Slot T5 & $-S_{2}^{*}$ & $S_{1}^{*}$ & $S_{4}^{*}$ & $S_{3}^{*}$ \\
Slot T6 & $-S_{3}^{*}$ & $S_{4}^{*}$ & $S_{1}^{*}$ & $-S_{2}^{*}$ \\
Slot T7 & $-S_{4}^{*}$ & $-S_{3}^{*}$ & $S_{2}^{*}$ & $S_{1}^{*}$ \\
\hline
\end{tabular}

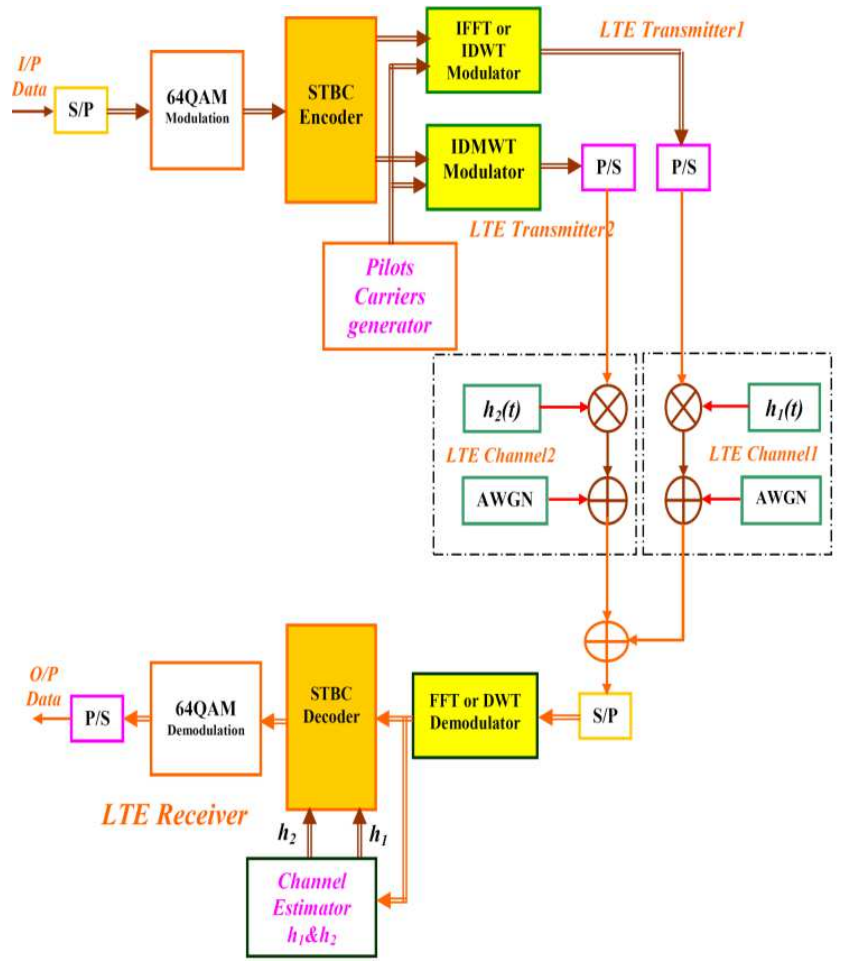

Fig. (1). Proposed Structure of STBC- LTE system

\section{The Specific LTE Channel Models}

The "Third Generation Partnership Project" 3GPP "Technical Recommendation" (TR) [3] defines three different type of multipath fading channel models: "the Extended Pedestrian A (EPA), Extended Vehicular A (EVA), and Extended Typical Urban (ETU)". All these channelmodeling functions will be used in this work examined the effect of these models. The higher-mobility profiles will not be used as "the closed-loop spatial-multiplexing mode". It is applicable to high data-rate and low-mobility scenarios only. These models enable the system to evaluate the performance of the proposed LTE transceiver in multichannel conditions reference. The model of any multipath fading channel can be defined by delay profiles and its relative power vectors. The maximum Doppler shift (MDS) or Doppler frequency must define with data rate in the channel model. The delay profiles of these models of channel define at low, medium, and high delay spread environment, respectively corresponding to (5, 70 , or $300 \mathrm{~Hz}$ ) as the maximum Doppler shift as shown in table (2) that clarify the channel delay profile of each model with values of tap delay (in nanoseconds) and relative power (in decibels).

In multi input multi output (MIMO) scenario of transmission, the spatial correlations between transmit and receive antennas are important factor that affect directly to the overall transceiver connection performance.

Table (2). LTE channel models (EPA, EVA, ETU) and delay profiles [3]

\begin{tabular}{lll}
\hline Channel model & Excess tap delay (ns) & Relative power (dB) \\
\hline Extended & {$[0,30,70,90,110,190$,} & {$[0,-1,-2,-3,-8$,} \\
Pedestrian A(EPA) & $410]$ & $-17.2,-20.8]$ \\
Extended Vehicular & {$[0,30,150,310,370$,} & {$[0,-1.5,-1.4,-3.6$,} \\
A(EVA) & $710,1090,1730,2510]$ & $-0.6,-9.1,-7,-12$, \\
Extended Typical & {$[0,50,120,200,230$,} & {$[-1,-1,-1,0,0,0$,} \\
Urban (ETU) & $500,1600,2300,5000]$ & $-3,-5,-7]$ \\
\hline
\end{tabular}

\section{Proposed STBC-LTE Systems Simulation Results}

In this section the proposed STBC-LTE DWT and FFT systems had been simulated using MATLAB 2014a. The BER performance of the proposed LTE system considered in different type of channel models mentioned above, the AWGN channel, the flat fading channel, and the selective multipath fading channel. The carrier frequency used in all these scenario was $2.3 \mathrm{GHz}$ to fixed and mobile proposed LTE system with three values of $M D S(5 \mathrm{~Hz}$ represented a mobility speed of $2 \mathrm{~km} / \mathrm{hr}, 70 \mathrm{~Hz}$ to speed $30 \mathrm{~km} / \mathrm{hr}$, and $300 \mathrm{~Hz}$ to speed $120 \mathrm{~km} / \mathrm{hr}$ ) to used LTE channels models Path Loss (Extended Pedestrian, Extended Vehicular, Extended Typical Urban) that was mentioned in section (3) for selective Multipath fading channel. Table (3) shows the parameters used to simulate the proposed LTE systems.

Table (3). Parameter of Simulation LTE system

\begin{tabular}{llllll}
\hline Parameter & $\begin{array}{l}\text { Fixed LTE } \\
\text { OFDM-PHY }\end{array}$ & \multicolumn{4}{l}{$\begin{array}{l}\text { Mobile LTE Scalable } \\
\text { OFDMA-PHY }\end{array}$} \\
\hline $\begin{array}{l}\text { Multicarrier size FFT } \\
\text { or DWT }\end{array}$ & 256 & 128 & 512 & 1024 & 2048 \\
$\begin{array}{l}\text { Number data used as } \\
\text { subcarriers }\end{array}$ & 96 & 64 & 180 & 360 & 720 \\
$\begin{array}{l}\text { Types of Modulation } \\
\text { Cyclic prefix or } \\
\text { guard band (Tg/Tb) }\end{array}$ & 64QAM & & & & \\
$\begin{array}{l}\text { Bandwidth of } \\
\text { Channel (MHz) }\end{array}$ & 20 & 20 & 20 & 20 & 20 \\
\hline
\end{tabular}

\subsection{STBC-LTE Performance in AWGN Channel}

As shown in the table (3), only the size of 256-subcarrier is used to (FFT or DWT) for fixed STBC-LTE. Also only AWGN model will be used to represent the channel model. The After simulation to all STBC-LTE system and collected the result of each system in Fig.(2). Also to compare the 
performance of these system, the $\mathrm{BER}=10^{-4}$ will be taken as a level of comparison. This figure shows that in FFT system reach the comparison level at SNR $38 \mathrm{~dB}$ for 1 antenna and SNR decreasing to $32 \mathrm{~dB}$ in 4 antenna while in DWT system the SNR is about $18 \mathrm{~dB}$ in 1 antenna and decreasing to $8 \mathrm{~dB}$ in 4 antenna, so a gain of $6 \mathrm{~dB}$ due to use STBC in FFT systems and $10 \mathrm{~dB}$ in DWT system because of multiple antennas in transmitter side will enhance the spectral efficiency of LTE system and improve better error rate and these benefits come with no extra cost in power and little lost in bandwidth. Also from the same figure, it is shown clearly that the proposed DWT based STBC-LTE is much better than the FFT based STBC-LTE with a gain of $20 \mathrm{~dB}$, so with using of 4 transmitters with STBC-LTE, again of $30 \mathrm{~dB}$ can be achieved. These results confirm that the DWT orthogonal base is more significant than the FFT orthogonal bases.

The size of $(128,512,1024,2048)$ to (FFT or DWT) For mobile LTE used are as shown in table (3) .Generally the performance of BER decrease with the increasing of the subcarriers size as shown in fig.(3), fig.(4), fig.(5) and fig.(6). And also the idea of using STBC will decrease the BER and enhance the performance of system. And using of DWT instead of FFT will enhance the performances of proposed system.

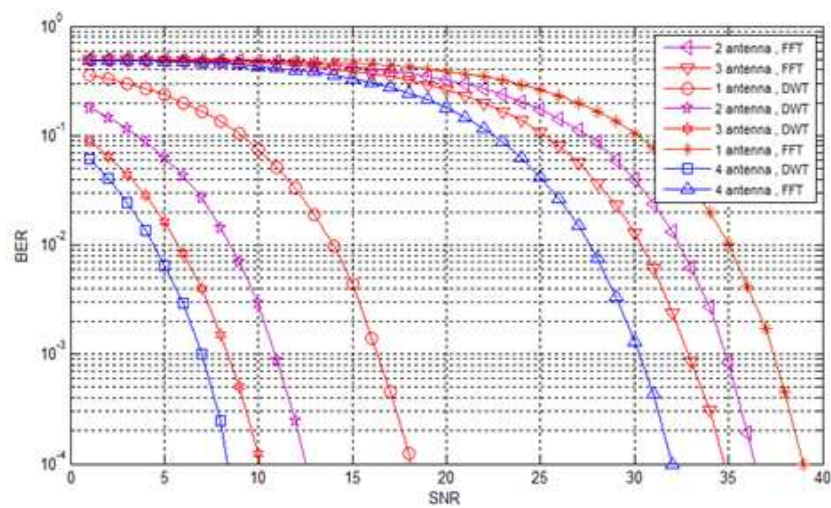

Fig. (2). SNR Versus BER to Fixed STBC-LTE-256 subcarriers in AWGN channel model

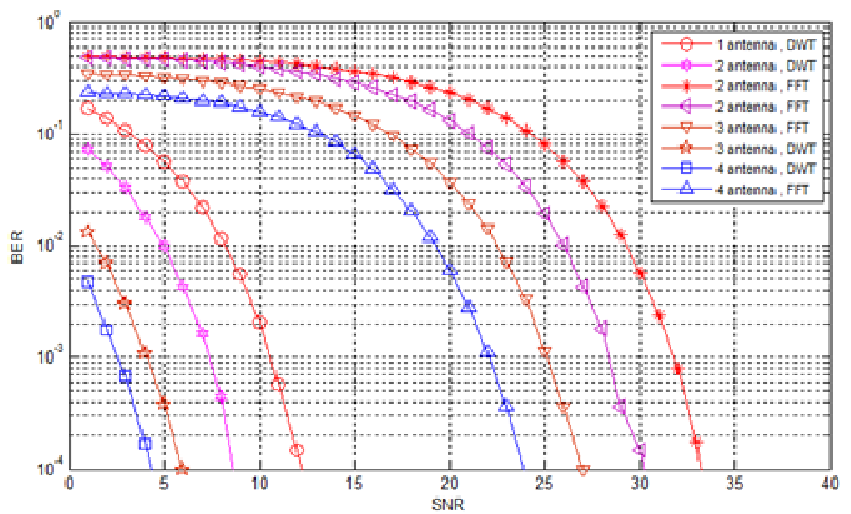

Fig. (3). SNR Versus BER to Mobile STBC-LTE-128 subcarriers in AWGN channel model

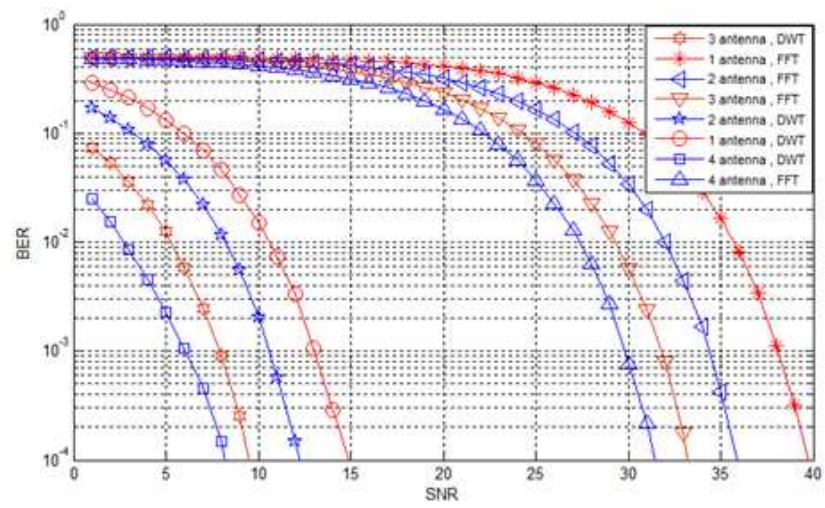

Fig. (4). SNR Versus BER to Mobile STBC-LTE-512 subcarriers in AWGN channel model

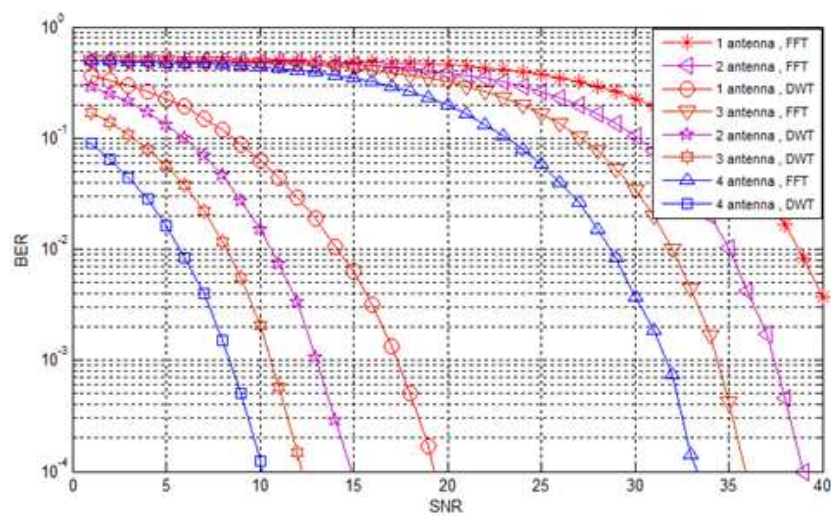

Fig. (5). SNR Versus BER to Mobile STBC-LTE-1024 subcarriers in AWGN channel model.

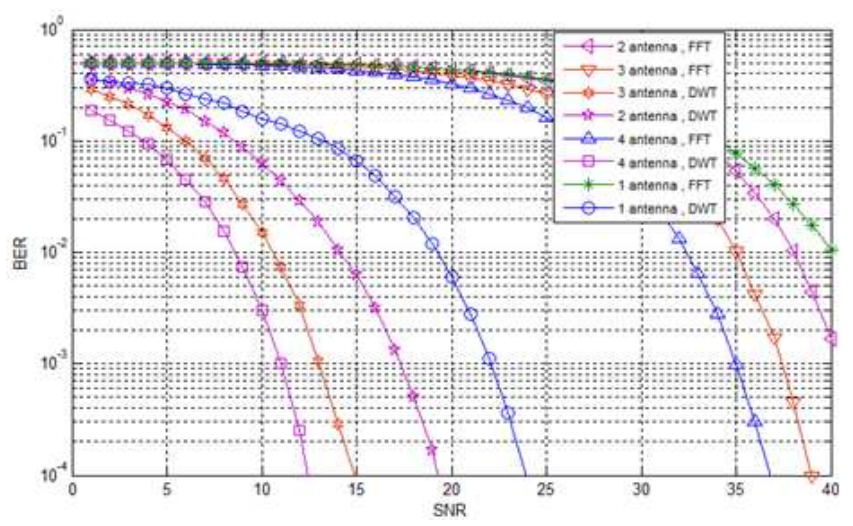

Fig. (6). SNR Versus BER to Mobile STBC-LTE-2048 subcarriers in AWGN channel model

\subsection{STBC-LTE Performance in Flat Fading Channel}

The same proposed STBC-LTE will examine by flat fading model in addition to AWGN. So the transmitted signals will be suffer from a constant attenuation and linear phase distortion through MIMO channels. The model that will represent flat fading channel will be the Rayleigh's distribution model. For fixed STBC-LTE system, the proposed system with STBC and DWT still performs better than the STBC-LTE based on FFT as shown in fig.(7). This figure shows that in FFT system reach the comparison level at SNR $39 \mathrm{~dB}$ for 1 antenna and SNR decreasing to $33 \mathrm{~dB}$ in 
4 antenna, while in DWT system the SNR is about $19 \mathrm{~dB}$ in 1 antenna and decreasing to $9 \mathrm{~dB}$ in 4 antenna, so a gain of $6 \mathrm{~dB}$ due to use STBC in FFT systems and 10dB in DWT system due to multiple antennas used in transmitters to enhance the system spectral efficiency and supports BER performance. These benefits come with no extra cost in power and little lost in in data rate and bandwidth. Also from the same figure, it is shown clearly that the proposed STBC-LTE based on DWT is much better than the STBC-LTE based on FFT with a gain of $20 \mathrm{~dB}$, so with using of 4 transmitters with STBCLTE, again of $30 \mathrm{~dB}$ can be achieved.

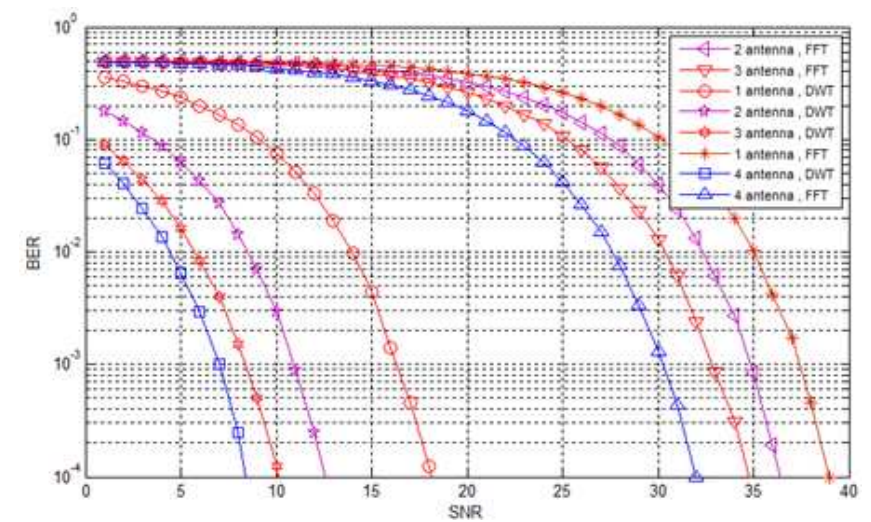

Fig. (7). SNR Versus BER to Fixed STBC-LTE-256 subcarriers in model of flat fading Channel

The simulation had been done in values of the Doppler frequencies $(M D S)$ of $5 \mathrm{~Hz}, 70 \mathrm{~Hz}$ and $300 \mathrm{~Hz}$ for mobile system that correspond to speed between the transmitter and receiver about 2,30 and $120 \mathrm{~km} / \mathrm{h}$ respectively. In all subcarriers sizes, a smaller effect in BER performance appears in $M D S=5 \mathrm{~Hz}$ while the larger effect appears in high Doppler frequency $M D S=300 \mathrm{~Hz}$ as shown in fig.(8),(9) and (10) for subcarrier 128. And fig.(11),(12) and (13) for subcarrier 512. And fig.(14),(15) and (16) for subcarrier 1024. And fig.(17),(18) and (19) for subcarrier 2048. Also it is clear from all simulation results that proposed system STBC-LTE with DWT perform better performance the STBC-LTE based on FFT in all Doppler frequencies used in model of flat fading Channel.

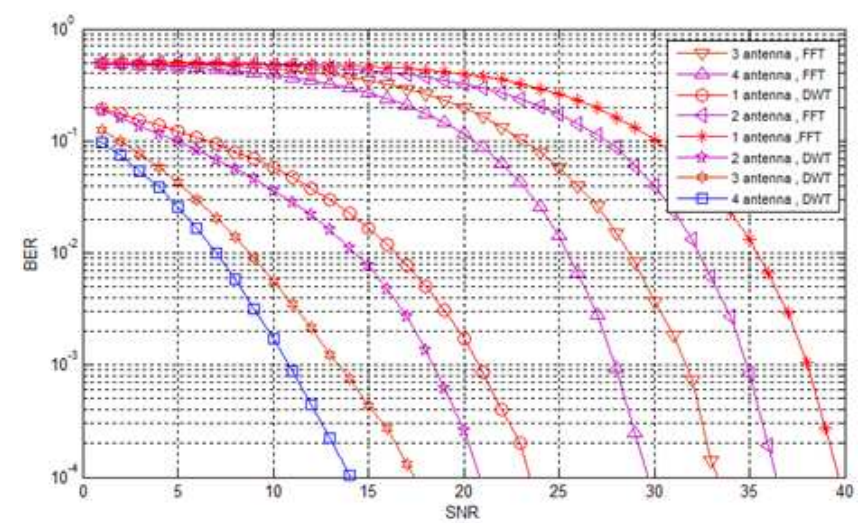

Fig. (8). SNR Versus BER to Mobile STBC-LTE-128 subcarriers in model of flat fading Channel-MDS=5 $\mathrm{Hz}$

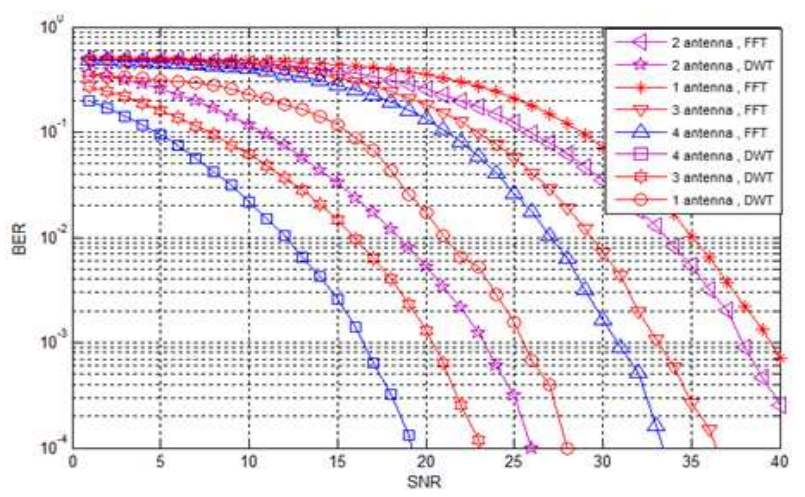

Fig. (9). SNR Versus BER to Mobile STBC-LTE-128 subcarriers in model of flat fading Channel $-\mathrm{MDS}=70 \mathrm{~Hz}$

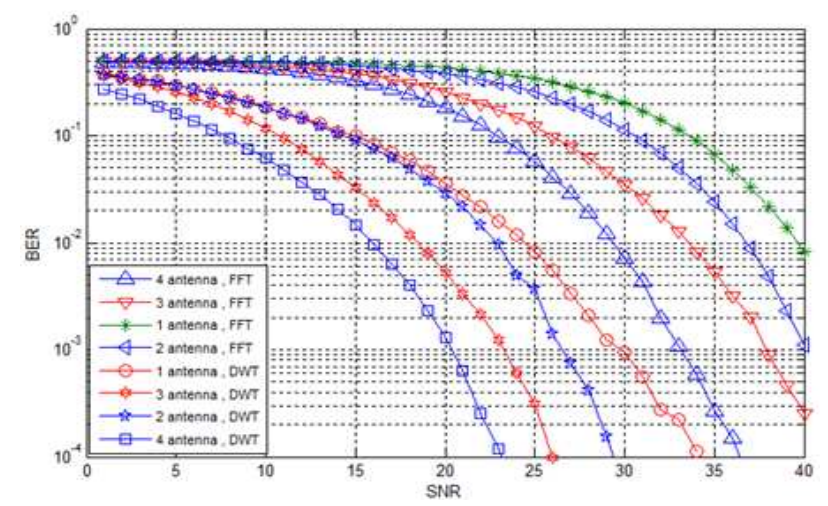

Fig. (10). SNR Versus BER to Mobile STBC-LTE-128 subcarriers in model of flat fading Channel $-M D S=300 \mathrm{~Hz}$

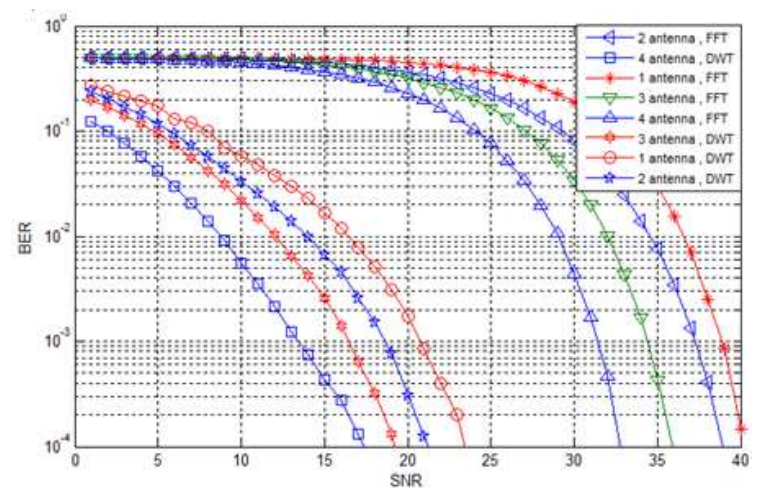

Fig. (11). SNR Versus BER to Mobile STBC-LTE-512 subcarriers in model of flat fading Channel $-M D S=5 \mathrm{~Hz}$

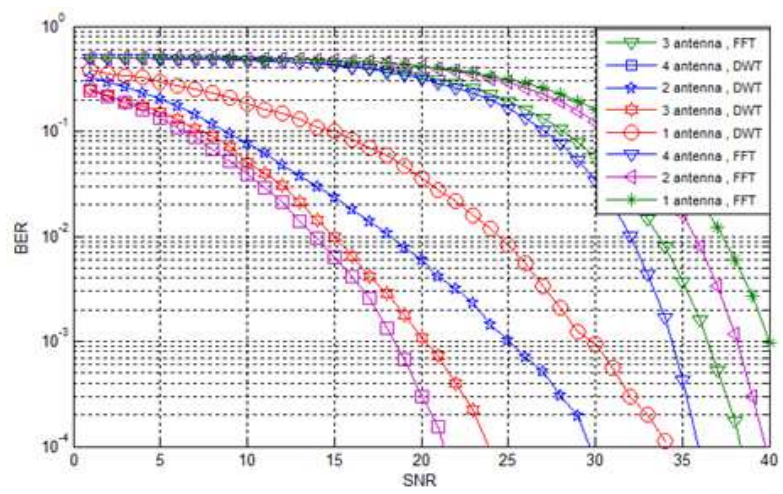

Fig. (12). SNR Versus BER to Mobile STBC-LTE-512 subcarriers in model of flat fading Channel $-\mathrm{MDS}=70 \mathrm{~Hz}$ 


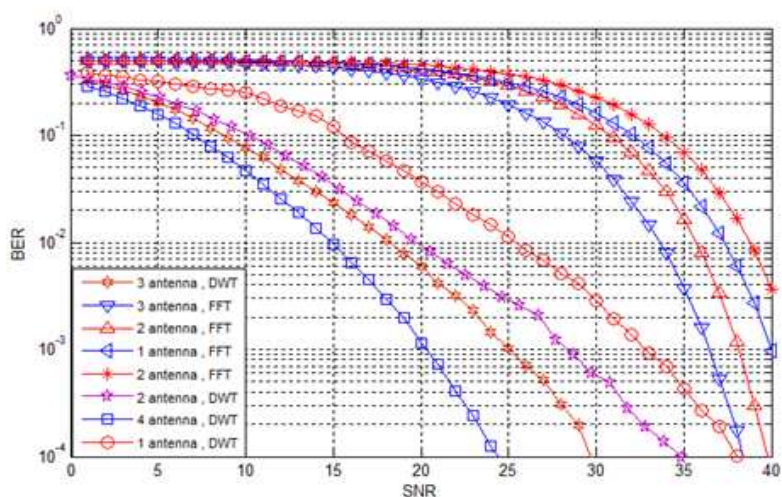

Fig. (13). SNR Versus BER to Mobile STBC-LTE-512 subcarriers in model of flat fading Channel $-\mathrm{MDS}=300 \mathrm{~Hz}$

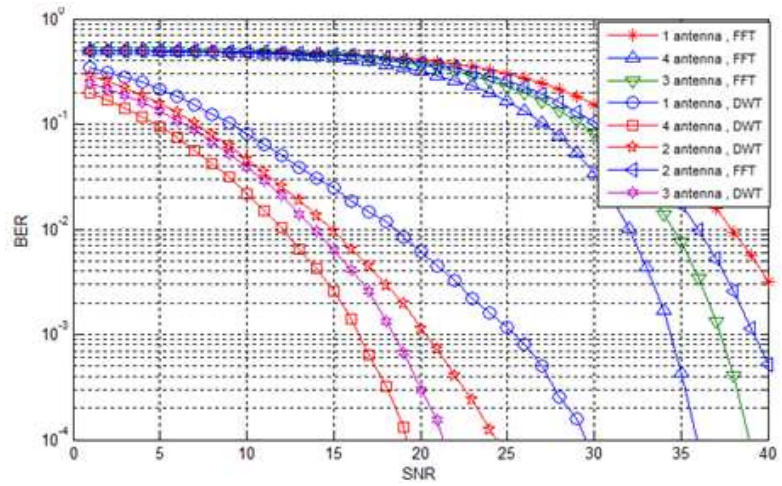

Fig. (14). SNR Versus BER to Mobile STBC-LTE-1024 subcarriers in model of flat fading Channel $-\mathrm{MDS}=5 \mathrm{~Hz}$

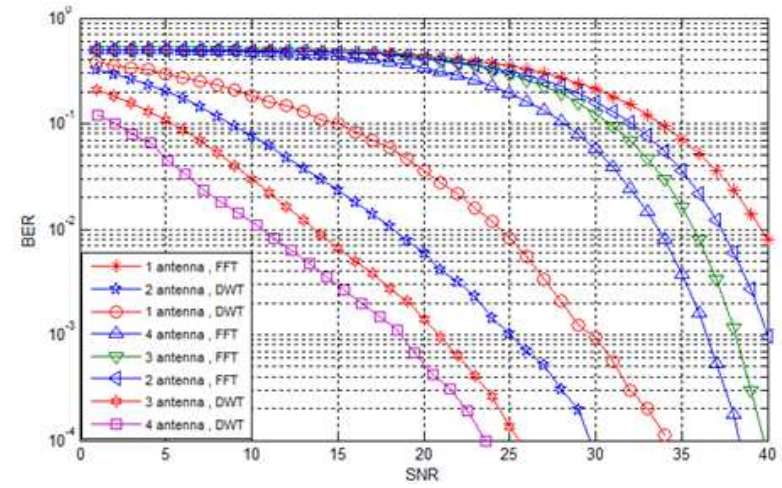

Fig. (15). SNR Versus BER to Mobile STBC-LTE-1024 subcarriers in model of flat fading Channel $-M D S=70 H z$

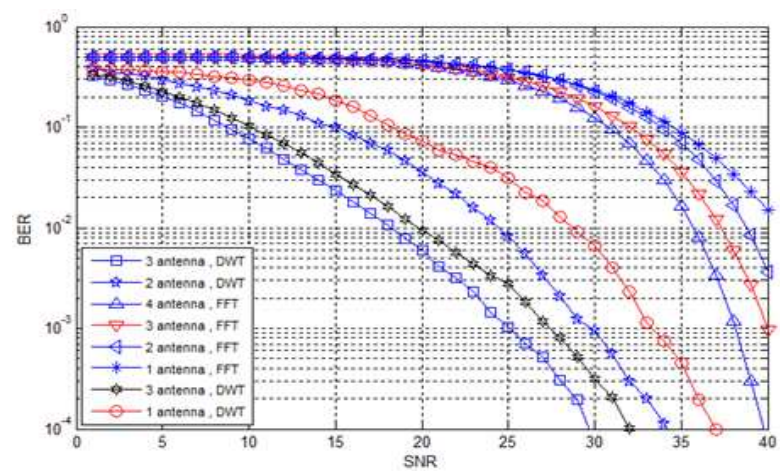

Fig. (16). SNR Versus BER to Mobile STBC-LTE-1024 subcarriers in model of flat fading Channel $-\mathrm{MDS}=300 \mathrm{~Hz}$

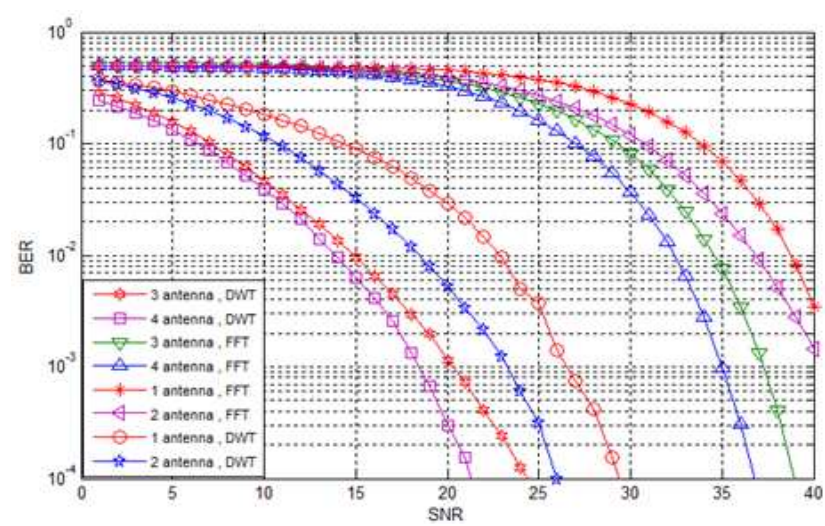

Fig. (17). SNR Versus BER to Mobile STBC-LTE-2048 subcarriers in model of flat fading Channel $-M D S=5 \mathrm{~Hz}$

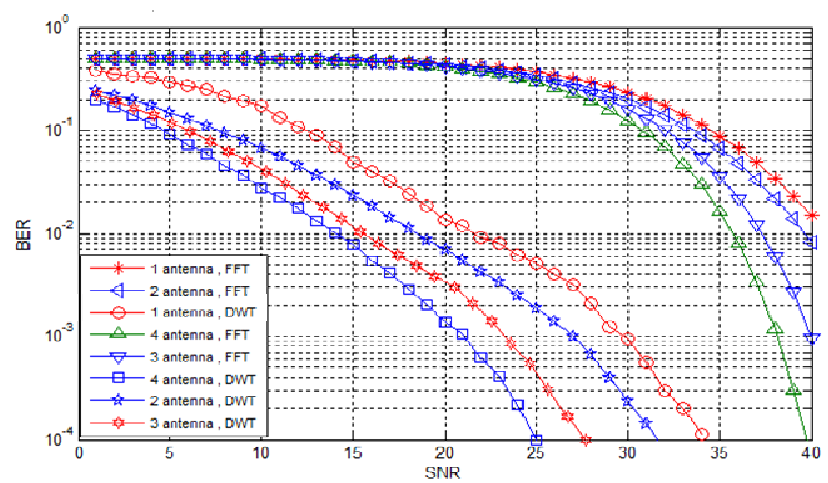

Fig. (18). SNR Versus BER to Mobile STBC-LTE-2048 subcarriers in model of flat fading Channel $-\mathrm{MDS}=70 \mathrm{~Hz}$

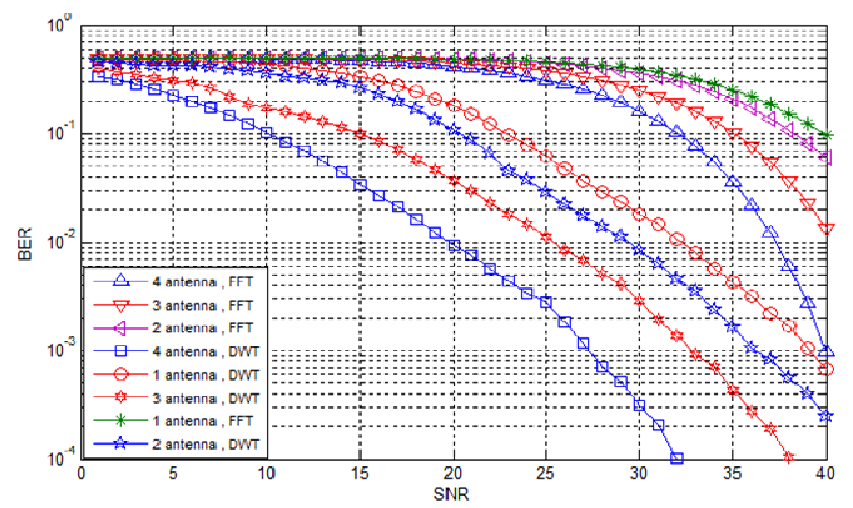

Fig. (19). SNR Versus BER to Mobile STBC-LTE-2048 subcarriers in model of flat fading Channel $-\mathrm{MDS}=300 \mathrm{~Hz}$

\subsection{STBC-LTE Performance in Multipath Fading Channel}

The same proposed STBC-LTE system shown in fig.(1) will examine by multipath selective Fading Channel model in addition to AWGN. So the transmitted signals will be suffer from multipath a constant attenuation and nonlinear phase distortion through MIMO channels. The model that will represent selective flat fading channel will be the Rayleigh's distribution model. All these model would been examining in three models of multipath fading channel: "the Extended Pedestrian A (EPA), Extended Vehicular A (EVA), and Extended Typical Urban (ETU)". In these types of channel, the transmitted signals frequency components are affected by 
uncorrelated changes corresponding to multipath. The Line of Sight (LOS) is one of them and the others paths are the reflected paths.

\subsubsection{Extended Pedestrian A (EPA) Channel Model}

The model of this channel was shown in table (1) first raw. The simulation had been done to all scenario of STBC-LTE with DWT and FFT and the simulation result shown in fig.(20) to fig.(26). In fixed STBC-LTE system it is noted that the proposed STBC-LTE based on DWT performs better than STBC-LTE based on FFT. The SNR at a BER $10^{-4}$ is about $19 \mathrm{~dB}$ for STBC-LTE based on DWT with 4 antennas and about $37 \mathrm{~dB}$ for STBC-LTE based on FFT with also using 4 antennas. A gain of about $18 \mathrm{~dB}$ has been achieved by using DWT way over the using FFT system and this value is much need in the communications systems to save transmitted power and increase data rate.

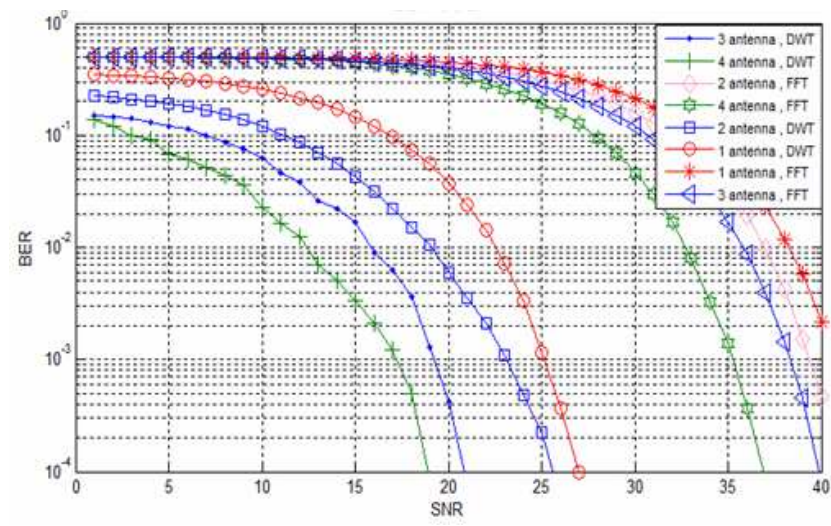

Fig. (20). SNR Versus BER to Fixed STBC-LTE-256 subcarriers in Extended Pedestrian A (EPA) Channel model

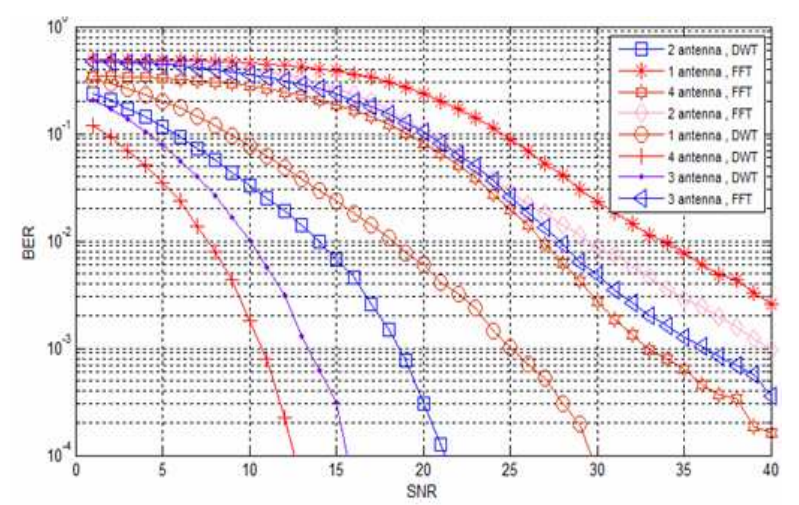

Fig. (21). SNR Versus BER to Mobile STBC-LTE-128 subcarriers in Extended Pedestrian A (EPA) Channel model - $M D S=5 \mathrm{~Hz}$

For mobile STBC-LTE system, the results of 128 and 1024 subcarriers (the size of DWT or FFT) only have been found in three cases of $M D S(5 \mathrm{~Hz}, 70 \mathrm{~Hz}$ and $300 \mathrm{~Hz})$. It can be seen from fig. (21) and fig. (22) (for small $M D S=5 \mathrm{~Hz}$ ) that the proposed STBC-LTE based on DWT still performs better than STBC-LTE based on FFT and the system of STBC still gives good results for small and large subcarriers (128 and 1024 respectively). The SNR at a BER $10^{-4}$ is about $12 \mathrm{~dB}$ for 4 antennas in proposed system of 128 and about $17 \mathrm{~dB}$ in 1024, while it's not reach the desired value in STBC-LTE based on FFT with 128 and 1024 subcarriers. In addition, a wide improvement span is obtained for all values of SNR in these systems.

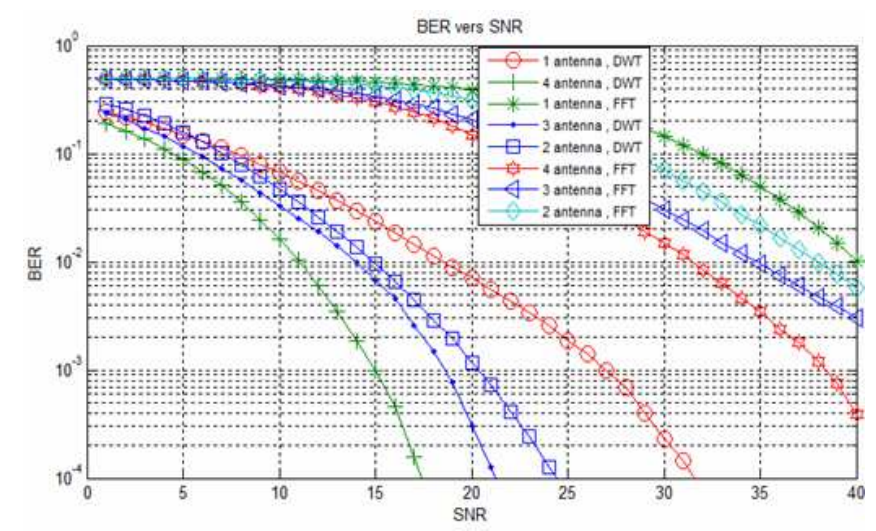

Fig. (22). SNR Versus BER to Mobile STBC-LTE-1024 subcarriers in Extended Pedestrian A (EPA) Channel model - $M D S=5 \mathrm{~Hz}$

It can be seen from figures (23), (25) for $70 \mathrm{~Hz}$ and (24), (26) for $300 \mathrm{~Hz}$ that the STBC- LTE based on DWT is performing better than the STBC-LTE based on FFT but without STBC, LTE based on DWT is better because of the effect of STBC is eliminated in high Doppler frequency larger than $50 \mathrm{~Hz}$ and the same for other systems .

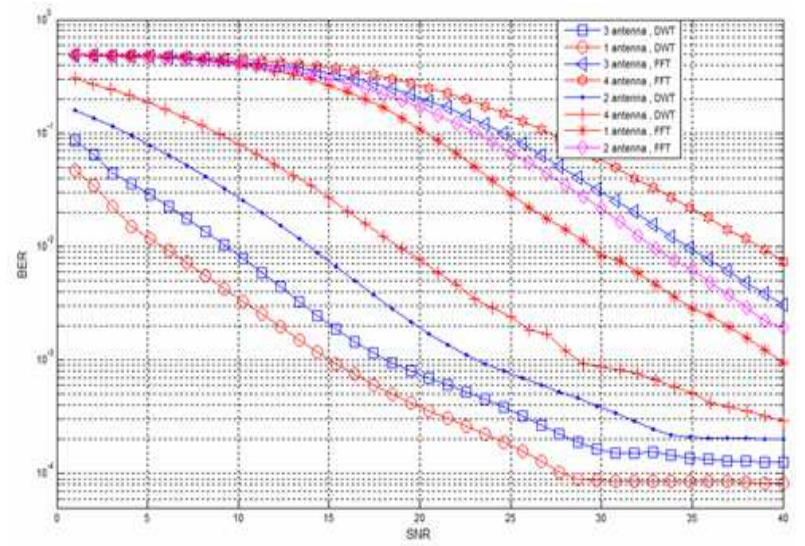

Fig. (23). SNR Versus BER TO Mobile STBC-LTE-128 subcarriers in Extended Pedestrian A (EPA) Channel model - MDS=70Hz

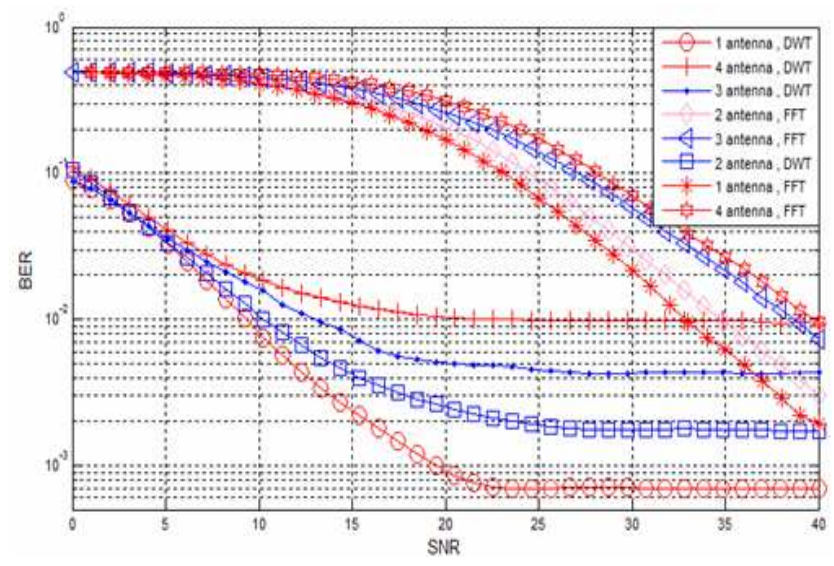

Fig. (24). SNR Versus BER to Mobile STBC-LTE-128 subcarriers in Extended Pedestrian A (EPA) Channel model - MDS=300Hz 


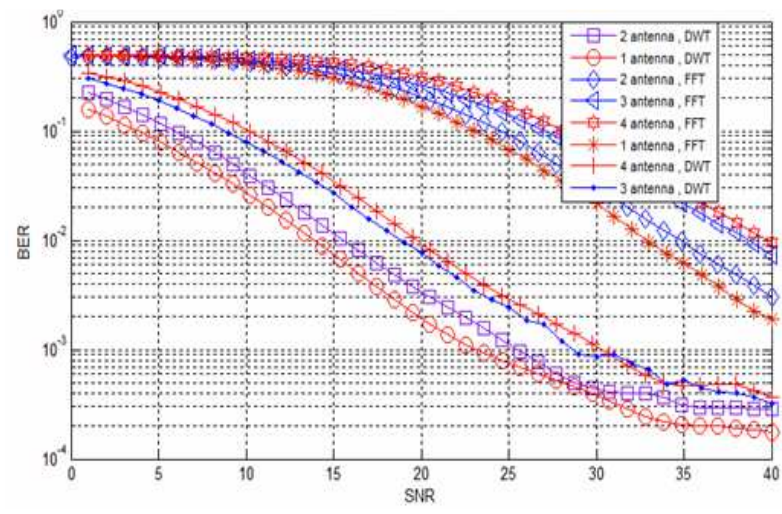

Fig. (25). SNR Versus BER to Mobile STBC-LTE-1024 subcarriers in Extended Pedestrian A (EPA) Channel model - $M D S=70 \mathrm{~Hz}$

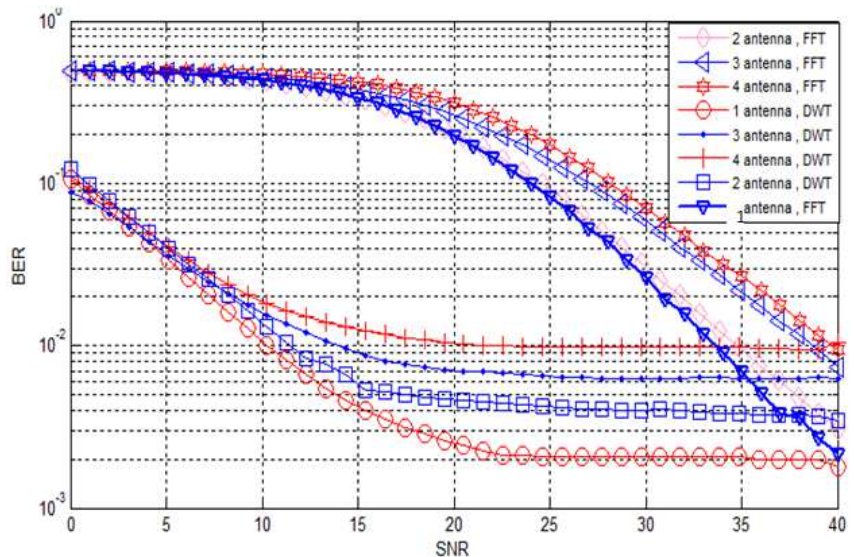

Fig. (26). SNR Versus BER to Mobile STBC-LTE-1024 subcarriers in Extended Pedestrian A (EPA) Channel model - MDS $=300 \mathrm{~Hz}$

\subsubsection{Extended Vehicular A (EVA) Channel Model}

In this section the results of Extended Vehicular A (EVA) channel model will be simulated according to the table (1) second raw. For fixed system, It is clear from Fig.(27) that BER performance of STBC-LTE based on DWT is better than the system of STBC - LTE based on FFT. The SNR at a $\mathrm{BER}=10^{-4}$ is about $21 \mathrm{~dB}$ for 4 antennas at proposed system and cannot been reached for STBC-LTE based on FFT system and this will give losses in the gain about $20 \mathrm{~dB}$ for proposed system against STBC-LTE with FFT system when compared with Extended Vehicular A (EVA) channel model.

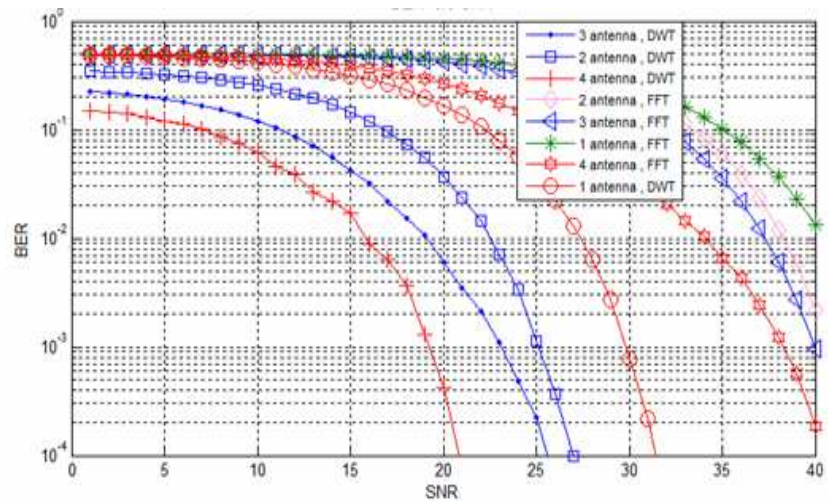

Fig. (27). SNR Versus BER to Fixed STBC-LTE-256 subcarriers in Extended Vehicular A (EVA) Channel model
Also for mobile system, the results have simulated only for 128 and 1024 (the size of DWT or FFT) in three cases of $M D S=(5 \mathrm{~Hz}, 70 \mathrm{~Hz}$ and $300 \mathrm{~Hz})$.

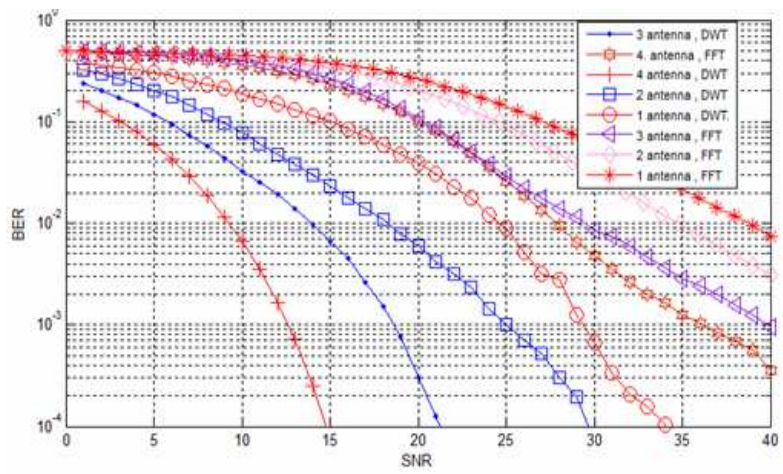

Fig. (28). SNR Versus BER to Mobile STBC- LTE-128 subcarriers in Extended Vehicular A (EVA) Channel model - MDS $=5 \mathrm{~Hz}$

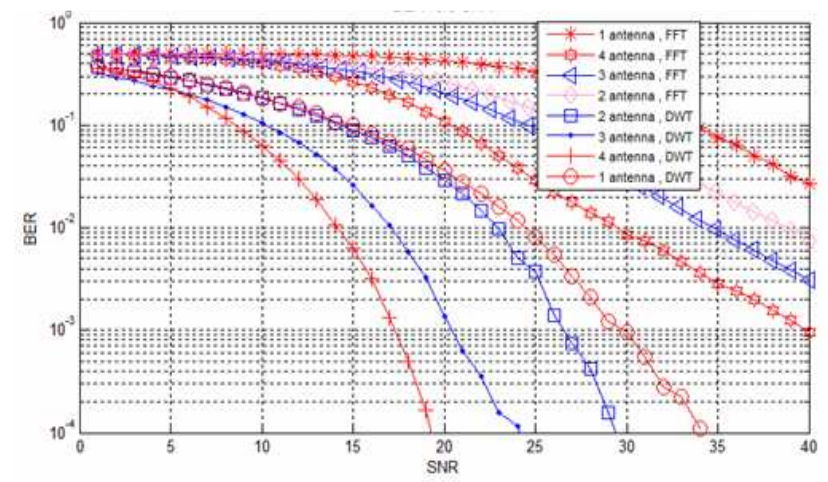

Fig. (29). SNR Versus BER to Mobile STBC-LTE-1024 subcarriers in Extended Vehicular A (EVA) Channel model - MDS $=5 \mathrm{~Hz}$

From figure (28) and (29), it can be seen that the proposed DWT based STBC-LTE still performs better than FFT based STBC-LTE and the system of STBC still gives good results. The SNR at a BER $10^{-4}$ is about $15 \mathrm{~dB}$ for 4 antennas at proposed system of 128 and about $19 \mathrm{~dB}$ for 1024, while it is cannot been reach in FFT based STBC-LTE system of 128 and 1024 , this means losses in gain is about $20 \mathrm{~dB}$ for the proposed STBC-LTE system and cannot been reach for the traditional system in comparison with the Extended Vehicular A (EVA) channel model at MDS of $5 \mathrm{~Hz}$.

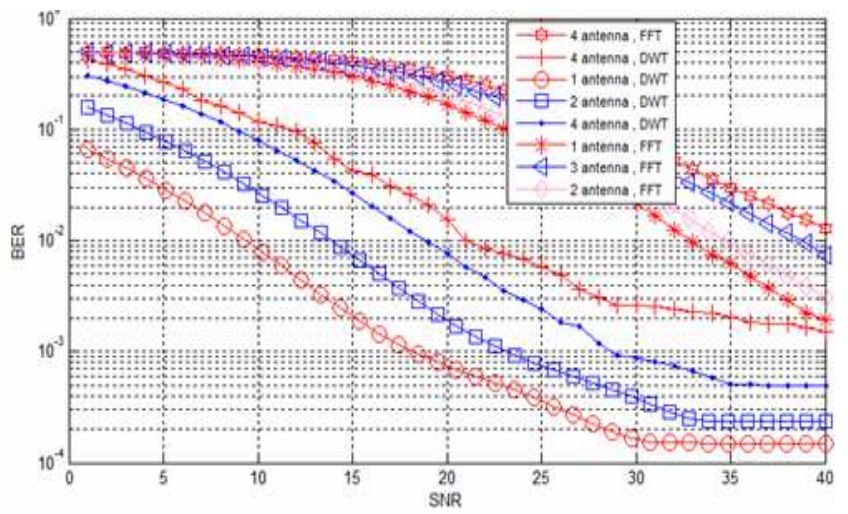

Fig. (30). SNR Versus BER to Mobile STBC-LTE in Extended Vehicular A (EVA) Channel model -128 subcarriers $-M D S=70 H z$ 


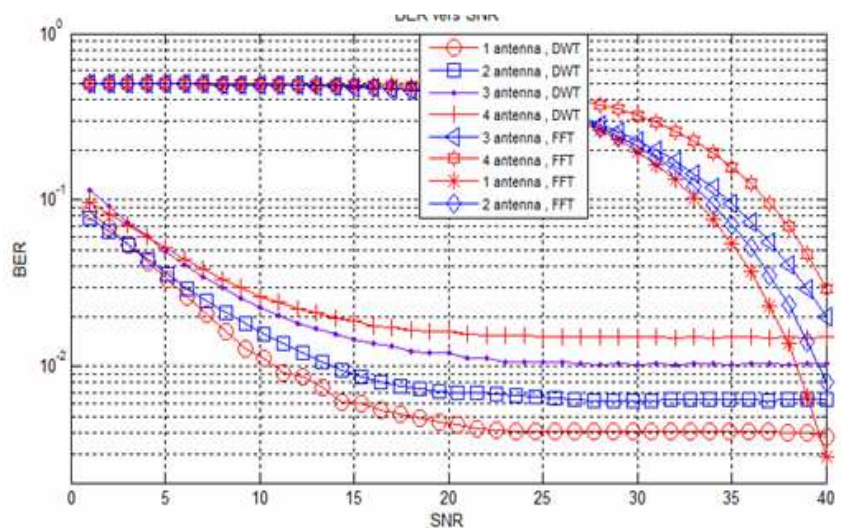

Fig. (31). SNR Versus BER to Mobile STBC-LTE in Extended Vehicular A (EVA) Channel model -128 subcarriers $-M D S=300 H z$

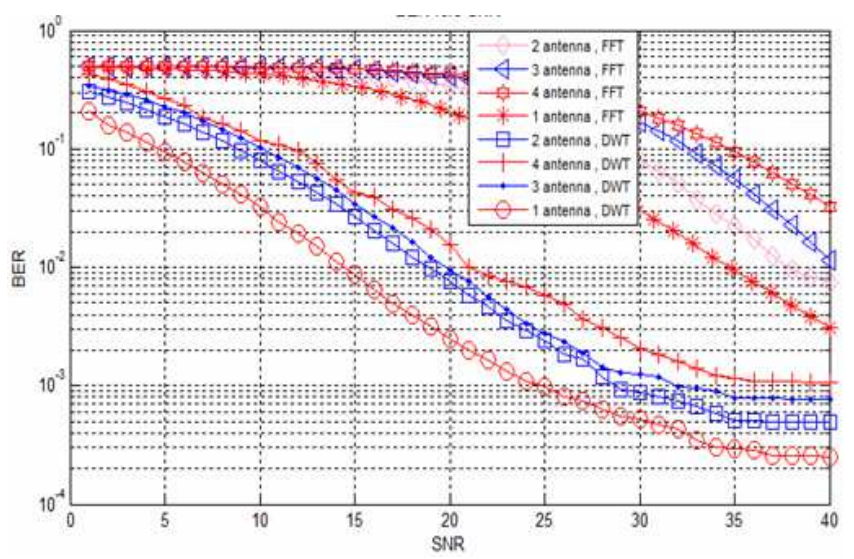

Fig. (32). SNR Versus BER to Mobile STBC-LTE-1024 subcarriers in Extended Vehicular A (EVA) Channel model - $M D S=70 H z$

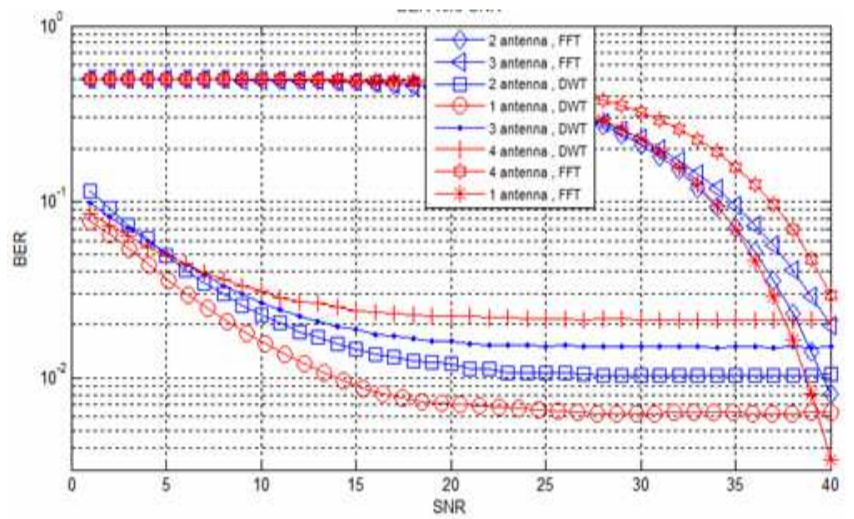

Fig. (33). SNR Versus BER to Mobile STBC-LTE-1024 subcarriers in Extended Vehicular A (EVA) Channel model - MDS $=300 \mathrm{~Hz}$

It can be seen from figures above that the losses will be increased for both systems due to Doppler Effect.

\subsubsection{Extended Typical Urban (ETU) Channel Model}

In this section the results of Extended Typical Urban channel model for vehicular test environment will be achieved. In this case the results will be worse than two other channel models because there are six cases with higher relative delay. For fixed LTE, It can see that the proposed DWT based STBC-LTE still performs better than FFT based STBC-LTE. The SNR at a BER $10^{-4}$ is about $21 \mathrm{~dB}$ for 4 antennas at the proposed system and non at FFT based STBC-LTE.

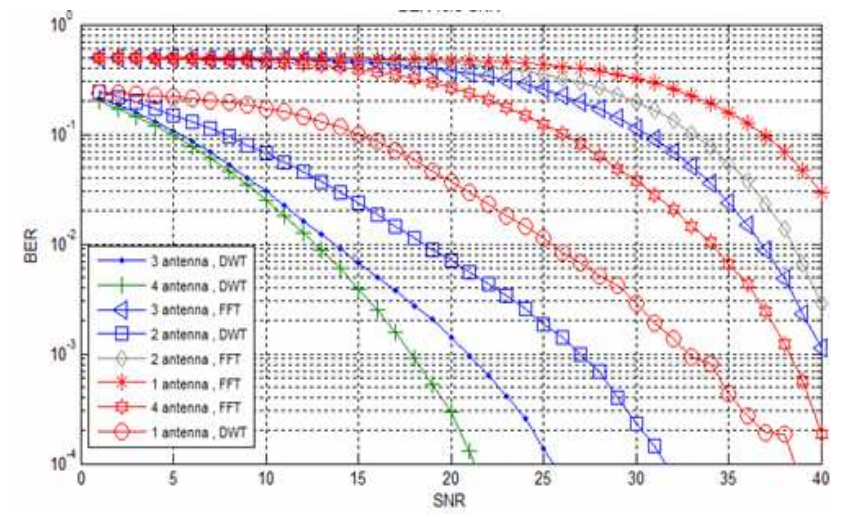

Fig. (34). SNR Versus BER to Fixed STBC-LTE-256 subcarriers in Extended Typical Urban (ETU) Channel model

For mobile system, the effect of MDS will appear and will directly affect on the system of STBC in the case of MDS higher than $50 \mathrm{~Hz}$ and the BER will increase as the Doppler frequency increases in both models. This will lead to results worse than the results of Extended Typical Urban (ETU) Channel model as display in the below figures.

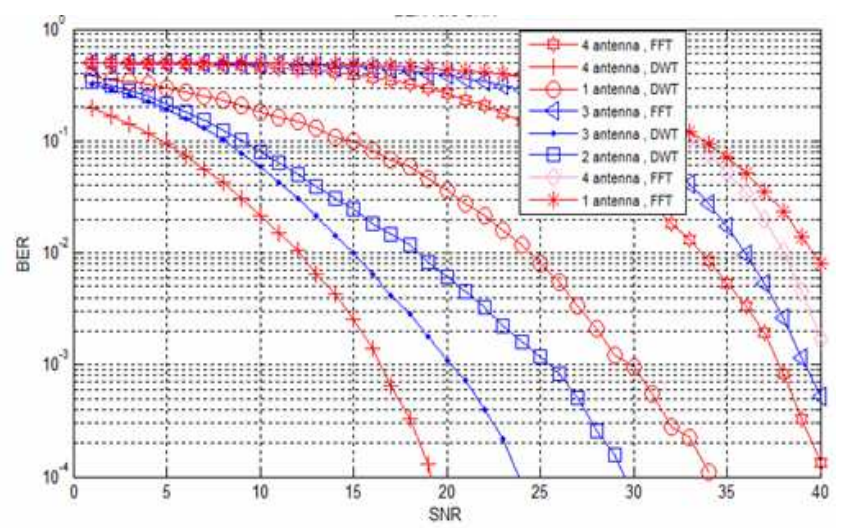

Fig. (35). SNR Versus BER to Mobile STBC- LTE-128 subcarriers in Extended Typical Urban (ETU) Channel model $-M D S=5 H z$

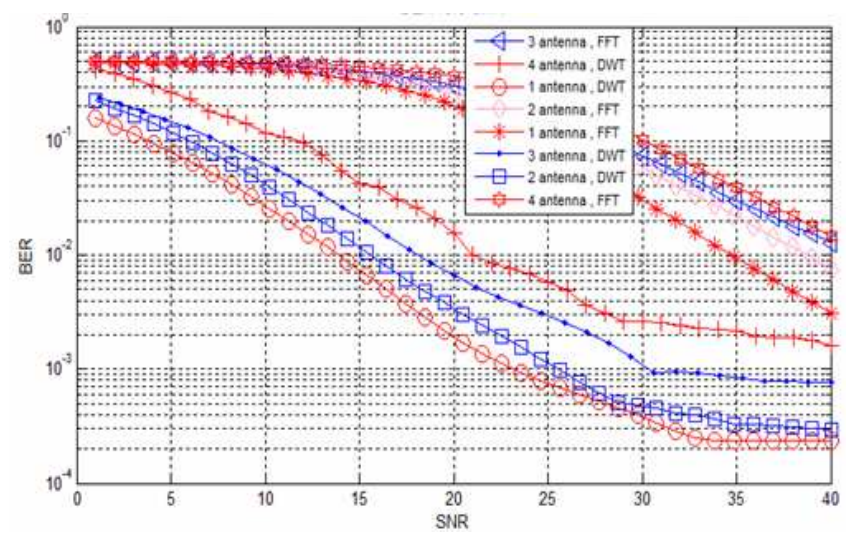

Fig. (36). SNR Versus BER to Mobile STBC-LTE-128 subcarriers in Extended Typical Urban (ETU) Channel model $-\mathrm{MDS}=70 \mathrm{~Hz}$ 


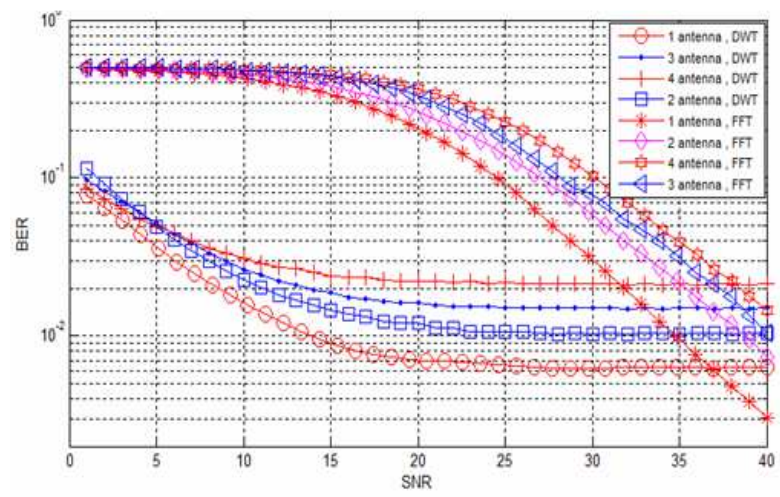

Fig. (37). SNR Versus BER to Mobile STBC-LTE-128 subcarriers in Extended Typical Urban (ETU) Channel model $-M D S=300 \mathrm{~Hz}$

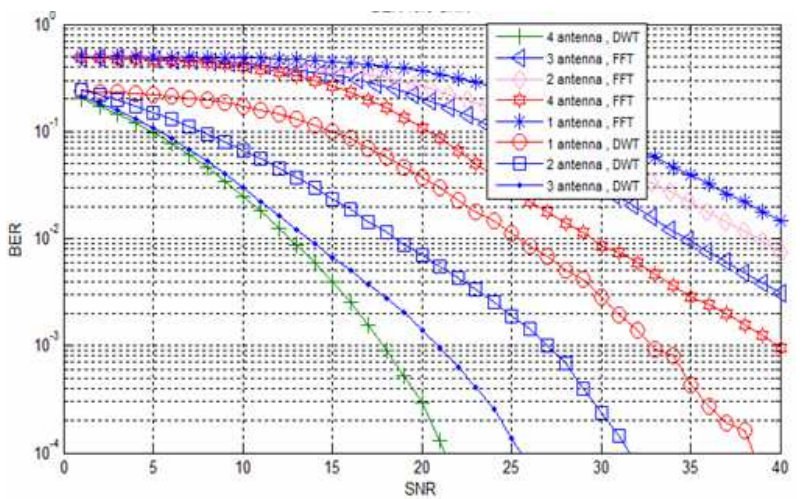

Fig. (38). SNR Versus BER to Mobile STBC-LTE-1024 subcarriers in Extended Typical Urban (ETU) Channel model $-\mathrm{MDS}=5 \mathrm{~Hz}$

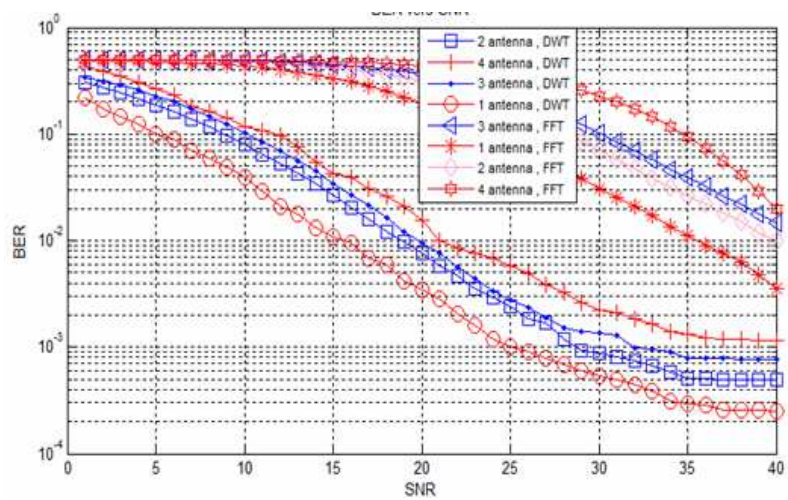

Fig. (39). SNR Versus BER to Mobile STBC-LTE-1024 subcarriers in Channel model $-\mathrm{MDS}=70 \mathrm{~Hz}$

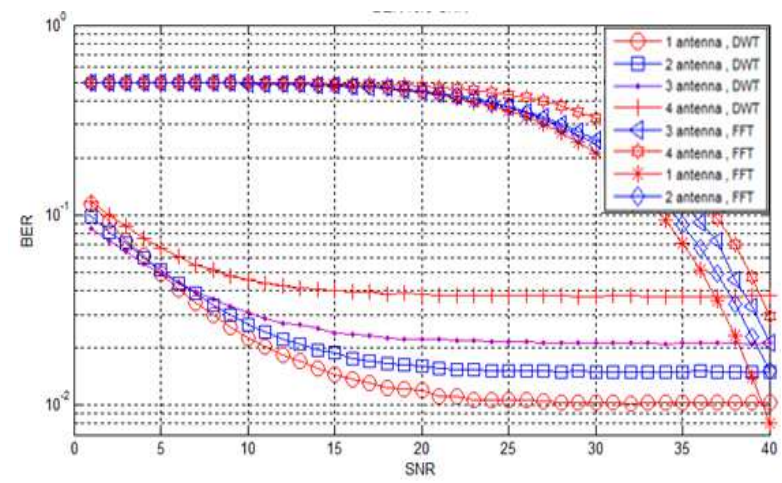

Fig. (40). SNR Versus BER to Mobile STBC-LTE-1024 subcarriers in Extended Typical Urban (ETU) Channel model $-M D S=300 \mathrm{~Hz}$
All the results present in paper computed after testing the system used transfer over $1 \mathrm{M}$ symbols.

\subsection{Discussion}

The BER performance of AWGN channel as show from the simulation results is the best of all channels as it has the lowest bit error rate (BER) and power losses with using 64QAM modulation schemes. The amount of noise occurs in the BER to this channel type is quite slighter than flat fading channels models. Simulations proved that the accumulation STBC and DWT improved the bit error rates BER performance than accumulation STBC and FFT in LTE system. The simulation results show that the worst scenario to all of the STBC- LTE systems, in terms of performance, occurs when the system parameter is changed. The STBCLTE with FFT shows that it requires a longer time adapting to noise and performs poorly to system parameter changes. It can be concluded from the comparison of the performance results of new STBC-LTE based DWT structure with the STBC-LTE based FFT that for the same model it gives a robust implementation and still performs better BER performance than FFT in all values of the Doppler frequencies model of flat fading Channel.

In the case of selective multipath fading channel, the simulation done in three cases of propagation losses model according to LTE channels models. Therefore it clear from the simulation results that DWT based STBC-LTE performs better than FFT based STBC-LTE ,but STBC advantage will be eliminated in high Doppler shift like at $70 \mathrm{~Hz}$ and $300 \mathrm{~Hz}$ because of each antenna attenuate each other and the losses in Extended Typical Urban (ETU) channel model is the worst case among other channel models Extended Pedestrian A (EPA), Extended Vehicular A (EVA), because of the combined effects of signal level attenuation in vehicular channel model due to the free space propagation, reflection, diffraction and scattering is more than those occurring in the other two channel models .

\section{Conclusion}

In this paper, first the companion of DWT and STBC in LTE structure has been designed and simulated for the first time. These simulations confirm successful operation and these structures are possibility of implementation. Also simulation results demonstrate BER performance enhancement that could achieve by combining the DWT and STBC techniques with very little decoding complexity to LTE system. As a result, the following points can be concluded:

1. It is clear that proposed STBC-LTE structure achieves much lower BER in AWGN, flat fading channel and selective Multipath fading channel. Therefore, this structure can be considered as an alternative to the conventional LTE system.

2. It is well known that the worst scenario to all of the STBC- LTE systems, in terms of performance, occurs when the system parameter is changed. The conventional scheme with FFT shows that it requires a longer time adapting to 
noise and performs poorly to system parameter changes. It can be concluded from the comparison of the performance of this new structure with the FFT that for the same model it gives a robust implementation.

3. In selective Multipath fading channels models, the simulation results are presented by isolating individual propagation effects, to discover which channel parameters have the most significant impact on the performance. In Doppler shift, it is seen that DWT based STBC- LTE still performs well better than FFT based STBC- LTE, but STBC advantage will be eliminated or lost in high Doppler shift above $50 \mathrm{~Hz}$ because of each antenna attenuate each other and the losses in Extended Typical Urban (ETU) channel model is the worst case among other channel models Extended Pedestrian A (EPA), Extended Vehicular A (EVA),.

\section{References}

[1] Baig S. and Mughal M. J., 2007 "A Frequency Domain Equalizer in Discrete Wavelet Packet Multitone Transceiver for In-Home PLC LANS", IEEE International Symposium on Power Line Communications and its Applications, March 2007, PISA, Italy.

[2] Fazelk and S. Kaiser-2002, "Multi-Carrier and Spread Spectrum Systems". 1st Edition, John Wiley \& Sons.

[3] Kitming, Tommy C.-2002, "Hybrids OFDM- CDMA: A Comparison of MC/DS-CDMA, MC-CDMA and OFCDM", Dept. of Electrical and Electronic, Adelaide University, SA 5005, Australia.

[4] Koga H., N. Kodama, and T. Konishi-2003, "High-speed power line communication system based on wavelet OFDM," in Proc. IEEE ISPLC 2003, Kyoto, Japan, May, pp. 226-231.

[5] Keita I., Daisuke U., and Satoshi D., 2007 "Performance Evaluation of Wavelet OFDM Using ASCET" IEEE.

[6] You-L. C. and Shiao-L. T., 2012 “A Low-Latency Scanning with Association Mechanism for Real-Time Communication in Mobile WiMAX", IEEE Transactions On Wireless Communications, Accepted For Publication.

[7] Qinghua Shi, Yong Liang Guan, Yi Gong and Choi Look Law, 2009 "Receiver Design for Multicarrier CDMA Using Frequency-Domain Oversampling", IEEE Transactions on Wireless Communications, Vol. 8, No. 5.

[8] Roberto C. and García A., 2009 "Joint Channel and Phase Noise Compensation for OFDM in Fast-Fading Multipath Applications", IEEE Transactions on Vehicular Technology, Vol. 58, No. 2.

[9] Sobia B., Gohar N.D., Fazal R., 2005 "An efficient wavelet based MC-CDMA transceiver for wireless communications", IBCAST
[10] Shun-Te Tseng and James S. Lehnert, 2009 "Windowing for Multicarrier CDMA Systems", IEEE Transactions on Communications, Vol. 57, No. 10

[11] Third Generation Partnership Project (3GPP), Dec. 2004 "Universal Mobile Telecommunications System (UMTS); Deployment aspects," (release 6), 3GPP. TS 25.943, version 6.0.0.

[12] Vasily S., Peter N., Gilbert S., Pankaj T., and Christopher H.1999, "The Application of Multiwavelet Filterbank to Image Processing", IEEE Transactions on Image Processing, Vol. 8, No. 4, pp(548-563),April.

[13] Yeen, Linnartz J-P and Fettweis G. -1993, "Multicarrier CDMA in Indoor Wireless Radio Networks". Proc. of IEEE PIMRC 1993,Yokohama, Japan, Sept. , pp.109-13

[14] Yu-Wei Lin, Hsuan-Yu Liu, and Chen-Yi Lee-2005 "A 1-GS/s FFT/IFFT Processor for UWB Applications" IEEE Journal of Solid-State Circuits, Vol. 40, No.8, pp.1726-1735.

[15] Yuan D., Zhang H., Jiang M. and Dalei Wu-2004 "Research of DFT-OFDM and DWT-OFDM on Different Transmission Scenarios." Proceedings of the 2nd International Conference on Information Technology for Application (ICITA), pp. 31-33.

[16] Zhang H., D. Yuan, M. Jiang and Dalei Wu-2004 "Research of DFT-OFDM and DWT-OFDM on Different Transmission Scenarios." Proceedings of the 2nd International Conference on Information Technology for Application (ICITA).

[17] Gupta A., Chandavarkar B. R.,2012, “An Efficient Bandwidth Management Algorithm for WiMAX (IEEE 802.16) Wireless Network EBM Allocation Algorithm.” IEEE Industrial and Information Systems (ICIIS).

[18] H. Zarrinkoub "Understanding LTE with MATLAB : from mathematical foundation to simulation, performance evaluation andimplementation” John Wiley \& Sons, Ltd,2014

[19] P. Tong, R. C. de Lamare, and A. Schmeink, "Adaptive distributed space-time coding based on adjustable code matrices for cooperative MIMO relaying systems," IEEE Trans. Commun., vol. 61, no. 7, pp. 2692-2703, Jul. 2013.

[20] Third Generation Partnership Project (3GPP), "Evolved Universal Terrestrial Radio Access (E-UTRA); Physical Channels and Modulation," standard specifications TS 36.211, 2009, available on www.3gpp.org, accessed on February 10, 2014.

[21] M. Suryanegara, and M.Asvial, "In Searching for 4G Mobile Service Applications: The Case of Indonesia Market," Telecommunications Journal of Australia, Vol.63, No.2, 2013.

[22] His-LuChao,Chia-kai Chang,Chia-Iung Liu, "A novel channel-aware frequency-domain scheduling in LTE uplink", Wireless Communication and Networking Conference (WCNC),2013 IEEE 